Scientific Paper

\title{
Dose assessment in high dose rate brachytherapy with cobalt-60 source for cervical cancer treatment: a phantom study
}

\author{
Bright Kwadwo BoUR ${ }^{a}$, Stephen INKOOM ${ }^{a, b, \star}$, Samuel Nii Adu TAGOE ${ }^{a, c}$, John Humphrey AMUASI ${ }^{a}$, Evans SASU ${ }^{c}$, \\ Francis HASFORD ${ }^{\mathrm{a}}$ \\ ${ }^{a}$ Department of Medical Physics, Graduate School of Nuclear and Allied Sciences, University of Ghana, Accra, Ghana \\ ${ }^{b}$ Radiation Protection Institute, Ghana Atomic Energy Commission, Accra, Ghana \\ ${ }^{c}$ Department of Radiotherapy, National Centre for Radiotherapy and Nuclear Medicine, Korle-Bu Teaching Hospital, Accra, \\ Ghana \\ *E-mail address: sinkoom@gmail.com
}

\begin{abstract}
Transition from low dose rate brachytherapy to high dose rate brachytherapy at our department necessitated the performance of dose verification test, which served as an end-to-end quality assurance procedure to verify and validate dose delivery in intracavitary brachytherapy of the cervix and the vaginal walls based on the Manchester system. An inhouse water phantom was designed and constructed from Perspex sheets to represent the cervix region of a standard adult patient. The phantom was used to verify the whole dose delivery chain such as calibration of the cobalt-60 source in use, applicator, and source localization method, the output of treatment planning with dedicated treatment planning system, and actual dose delivery process. Since the above factors would influence the final dose delivered, doses were measured with calibrated gafchromic EBT3 films at various points within the in-house phantom for a number of clinical implants that were used to treat a patient based on departmental protocol. The measured doses were compared to those of the treatment planning system. The discrepancies between measured doses and their corresponding calculated doses obtained with the treatment planning system ranged from -29.67 to $40.34 \%$ (mean of $\pm 13.27 \%$ ). These compared similarly to other studies.
\end{abstract}

Key words: brachytherapy; dose; gafchromic EBT3 films; Manchester system; in-house water phantom.

\section{Introduction}

Brachytherapy (BT) is an established radiotherapy treatment technique for the management of well-localized tumours. Brachytherapy makes use of miniaturized encapsulated radioactive sources that can be placed into or immediately adjacent to the tissue to be treated. This makes it possible to highly localize radiation dose to the tumour as well as tailoring the dose distribution to conform to the shape of the target. BT sources are designed such that the dose fall-off with distance within a tissue is very steep. This characteristic of BT sources facilitates the sparing of normal tissues in close proximity to the intended target from receiving a significant amount of radiation dose in contrast to external beam radiation therapy. For this reason, brachytherapy allows higher doses per fraction to be delivered to the tumour. ${ }^{1}$ BT implants are classified based on source placement, dose to a predefined location of the tumour within a specific time, and duration of BT treatment. For source placement, BT has many divisions, but the most widely used are interstitial brachytherapy (ISBT) and intracavitary brachytherapy (ICBT). In ISBT, radioactive sources are placed directly into the target tissue. For ICBT, the radiation sources are placed in a body cavity next to the tissue to be treated. In terms of dose to a predefined location of the tumour within a specific time, BT may be classified as lowdose-rate (LDR) or high-dose-rate (HDR) BT. ${ }^{2}$ Apart from these, there are hybrid forms of HDR BT tailored to produce the established biological effectiveness of LDR BT. ${ }^{3}$ The unique advantages of HDR BT over the LDR BT are that the former allows treatment on out-patient bases and better radiation dose optimization can be achieved. Nevertheless, HDR BT has the potential for accidental high exposures and execution of grievous errors which would have adverse effects on treatment outcome. There is therefore the need to institute quality assurance (QA) procedures for HDR BT in order to ensure safe treatment delivery and prevention of unplanned exposures. The major identified sources of error in any BT application are source calibration uncertainty and issues relating to source and organ localization. ${ }^{4}$ Additionally, BT treatment outcome is greatly influenced by the following: the particular model used for source distribution in the target volume, the algorithm used for calculation of dose distribution, methods used for the determination of the source strength, dose 
rate and prescribed dose used in treatment. Carefully taking cognizance of these processes and trying to minimize their effects on treatment outcome is very challenging and at times difficult to realize during treatment delivery. However, these challenges have been addressed by implementing in-vivo dosimetry (IVD) during BT treatment delivery. Miniaturised detectors are used to monitor easily accessible organs in close proximity to applicators placed within the body to hold sources. The high-gradient BT dose distribution and the large range of dose and dose rate pose challenges for most of the detectors in use for IVD in BT. Owing to these challenges, the sensitivity and specificity toward error detection have been limited, and IVD has mainly been restricted to the detection of gross errors.

The importance of assessing the performance of other detectors as well as other measurement techniques cannot be over-emphasized. In this study, an end-to-end QA test in HDR BT for the treatment of gynaecological cancers was instituted. A locally constructed water phantom to represent the pelvic region of an average adult human was used with calibrated Gafchromic EBT3 films to measure doses of critical structures (such as the bladder), which were compared to the calculated values from the treatment planning system (TPS) in use for BT treatment planning.

\section{Materials and Methods}

\section{Calibration of Gafchromic EBT3}

The Gafchromic EBT3 film (lot number: 04201601) which was used for dose measurements in the constructed cervix phantom, was calibrated against a $0.6 \mathrm{cc}$ Farmer type ionization chamber (TM 30001-01; SN: 00820; PTW Freiburg, Germany) using the International Atomic Energy Agency (IAEA) Technical Report Series (TRS) 398 protocol $^{6}$. Measurements to ensure effective calibration of the film for dose measurements were carried out in an MP1 one dimensional (1D) manual water phantom (PTW-Frieburg, Germany), using beams from an Equinox 100 Co-60 teletherapy machine (Best Theratronics, Canada). During the beam output measurement of the telecobalt machine, the ionization chamber was connected to a UNIDOS E electrometer (T10008-081102; PTW Freiburg, Germany), with the dosimetry system having traceability to a Secondary Standard Dosimetry Laboratory (SSDL), Chicago, United States of America.

During the Gafchromic film (Ashland Inc., USA) calibration to enable the film to be used for dose measurements, Gafchromic films from the same batch were cut into strips, each having dimensions of $7.8 \mathrm{~cm} \times 5.4 \mathrm{~cm}$, and irradiated to a known dose. In cutting the film strips, it was ensured that the longer side of a film strip corresponded with that of the large film from which a film strip was cut from. This was done to ensure that the strip films were scanned in the same direction in order to be used for the analysis process.

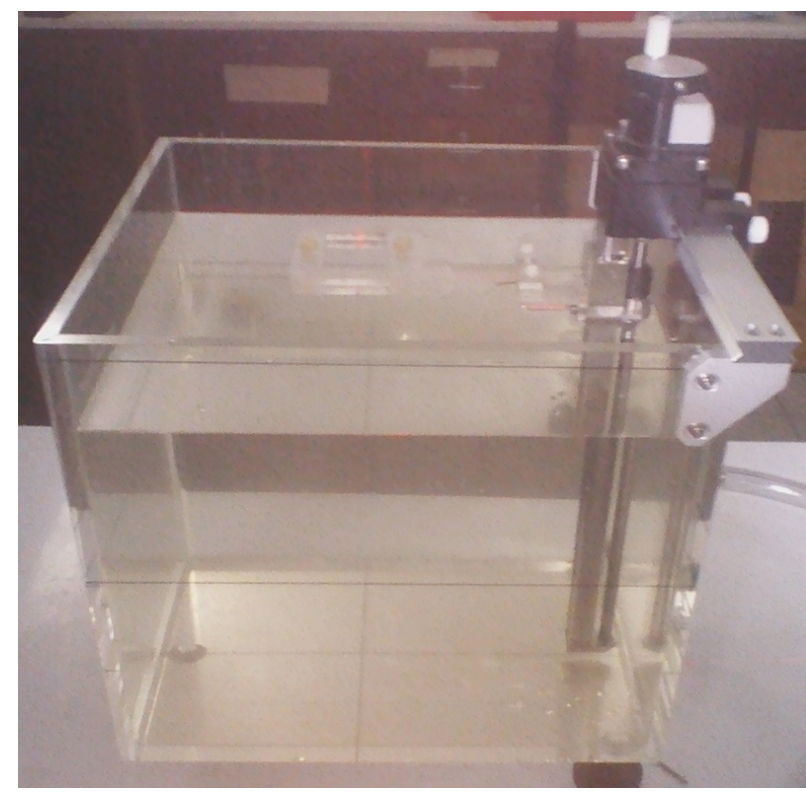

Figure 1. 1D manual water phantom with the locally fabricated film holder held at depth of $5 \mathrm{~cm}$ mounted on Equinox teletherapy couch

The film strips were irradiated with doses of: $0,2.8,5,6,7$, 9.4, 10, and 12 Gy using beams (each having field size of $10 \mathrm{~cm} \times 10 \mathrm{~cm}$ ) from the telecobalt machine. Source-to-surface distance (SSD) irradiation technique was employed. Treatment time for each dose was calculated from the beam output obtained for the telecobalt machine via the beam output measurements. For the film irradiations, the films were held at a depth of $5 \mathrm{~cm}$ in the 1D manual water phantom with the aid of a locally fabricated holder (Figure 1), such that a film was placed central to the radiation field.

A post-irradiation period of 3 days was allowed before exposed films were scanned in a batch with an Epson flatbed document scanner (CX5900; Epson, Australia), and scanned images were saved in tagged image file format (TIFF). The post-irradiation period was to facilitate stabilization of postexposure density growth ${ }^{7}$ prior to scanning and analyzing an exposed film. Images were acquired in reflective mode with the flatbed scanner. RGB-positive images were collected at a depth of 24 bits per colour channel with a spatial resolution of $72 \mathrm{dpi}$, and options were selected for the scanner not to apply any correction features to the scanned images. During the scanning process, all films were oriented with the longest dimension of the film perpendicular to the scanning direction. The scanned images were analyzed with ImageJ software (National Institutes of Health and the Laboratory for Optical and Computational Instrumentation, USA) to obtain optical densities of the exposed films. A region of interest (ROI) covering about one-third of the surface area of a film strip was used for the analyses. For each film, the ROI was placed central to the film during the optical density measurements. The reproducibility of the flatbed scanner was ascertained by scanning repeatedly a film at different times. Film nonuniformity and film-to-film variations were measured for six 
films (randomly selected) from the same film batch (or lot number), following the method proposed by Saur and Frengen. ${ }^{8}$ The overall accuracy of the EBT3 film measurements was derived using the method proposed by van Battum, ${ }^{9}$ which takes into account the most pronounced sources of uncertainties in dose determination (scanner response, lateral dependence correction, fit accuracy, intrabatch variation, background, intrinsic film inhomogeneity). For each colour channel (red, blue, and green), the correlation between the optical density of the exposed film and dose received by the film was determined using a graphical approach. The colour channel with the most sensitive and well correlated optical density to dose relationship was chosen as the calibration curve (sensitometric curve) for the film.

\section{Constructed Phantom}

A water tank with dimensions of $30 \mathrm{~cm} \mathrm{x} 30 \mathrm{~cm} \mathrm{x} 40 \mathrm{~cm}$ was constructed from Poly methyl methacrylate (PMMA) sheets with thickness of $0.6 \mathrm{~cm}$ for the study (Figure 2). A Perspex plate (PMMA), with thickness and length of $2.4 \mathrm{~cm}$ and $40 \mathrm{~cm}$, respectively, but with a width similar to the baseplate of the applicator clamp (LAZ 22-01; Eckert and Ziegler, Germany) in use for securing applicators for intracavitary gynaecological brachytherapy implants, was attached to one side of the constructed water tank. In attaching the Perspex plate to the water tank, it was done such that the length of the plate corresponded with the height of the tank. The other surface of the $2.4 \mathrm{~cm}$ thick Perspex plate had a groove to accommodate the base of the reconstruction box (LAZ 11-04; Eckert and Ziegler, Germany). The Perspex plate also had clamping devices made from Perspex for securing the baseplate of the applicator clamp. This Perspex plate serves as the base of the water tank. At the opposite side of this Perspex plate was a film holder mechanism designed to enable three-dimensional manual maneuvering of the detector. The film holder mechanism was positioned close to the mouth of the water tank. Adjacent to the film holder mechanism was a cradle, fabricated from Perspex sheets, placed at the bottom of the water tank to support the apex part of the reconstruction box. The other sides of the tank had handles for lifting the tank. The various parts of the constructed water tank were glued together with chloroform, which dissolves Perspex.

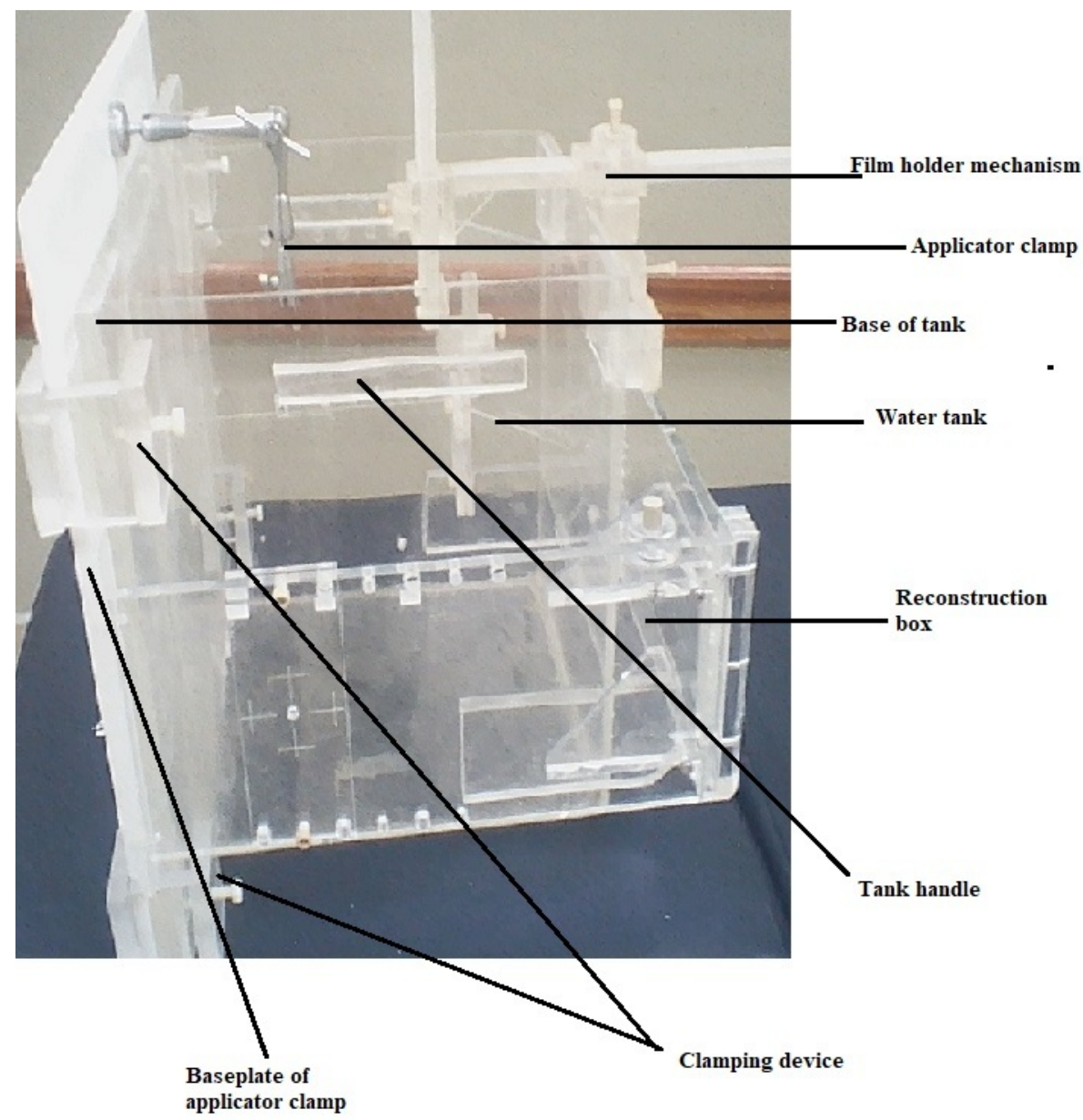

Figure 2. Constructed water phantom for gynaecological brachytherapy treatment simulations 


\section{Brachytherapy implant implementation with constructed phantom}

Prior to the simulation of brachytherapy treatment with the constructed phantom, QA tests were performed on the HDR brachytherapy afterloader machine (MultiSource HDR brachytherapy afterloader machine; Eckert and Ziegler, Germany). For the QA, the brachytherapy source was calibrated with a re-entrant well-type ionization chamber (HDR 1000 plus; Standard Imaging, USA) which was connected to a Max 4000 electrometer (Standard Imaging, USA), and the measured source strength compared to that of the TPS in use for brachytherapy treatment planning. The dosimetry system had traceability to an SSDL in Chicago, USA. Tests to check the accuracy of dwell position and dwell time were performed based on manufacturer recommendations.

After the QA procedures, the water tank phantom was placed on the brachytherapy table and filled with water close to the brim (Figure 3). The baseplate of the applicator clamp (LAZ 22-01) was attached to the water tank and secured in place with the clamping mechanism provided for the water tank. Before clamping the baseplate of the applicator clamp, the base of the reconstruction box was inserted into the groove on the base of the water tank, such that the base of the reconstruction box was central to the tank. The applicator clamp was therefore located at the mouth of the tank. The reconstruction box was mounted on its base and supported on the cradle of the water tank. Applicator configurations that would be used for patient treatment based on adopted departmental protocol were simulated with the water tank. Applicator configurations simulated were those for gynaecological brachytherapy treatments, such as: tandem and ovoids; for the treatment of cancer of the cervix, and tandem and cylinder; for the treatment of cancer of the endometrium. The departmental protocol is based on the Manchester system and Fletcher suite of applicators.

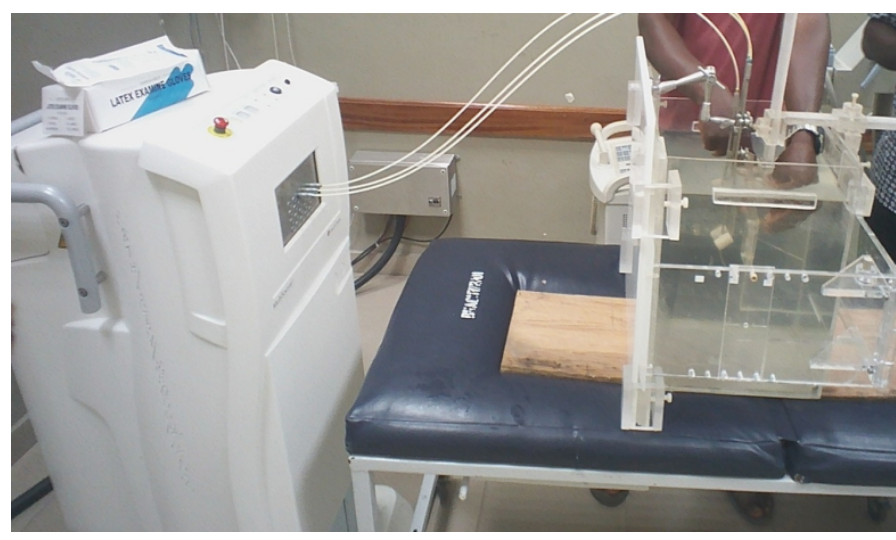

Figure 3. Phantom set up connected to afterloader machine with bladder marker being replaced with radiochromic film

For each implant, radiographs orthogonal to each other were taken with a mobile C-arm X-ray machine (Siemens, Germany) in a horizontal plane (perpendicular to the long axis of the water tank) to facilitate applicator and source localization during treatment planning. Figure 4 (A and B) shows the treatment set-up with the constructed phantom undergoing imaging. The set-up with the constructed phantom mimics a patient undergoing gynaecological brachytherapy standing. The applicators were held in the water tank with the aid of the applicator clamp as labeled in Figure 2, and doses to presumed bladder locations measured with Gafchromic EBT3 films selected from the same batch as those used for the film calibration. In this study, dose measurements were limited to the bladder, as this organ is not easily accessible in clinical treatments. The same size of the film strip as those used for the calibration was used for measurements. The films were held in the water with the aid of the film holder mechanism attached to the water tank. During imaging of an implant within the phantom with the $\mathrm{C}$-arm mobile X-ray machine, the location of the bladder was marked with a film strip having a small radioopaque object implanted at its central part.
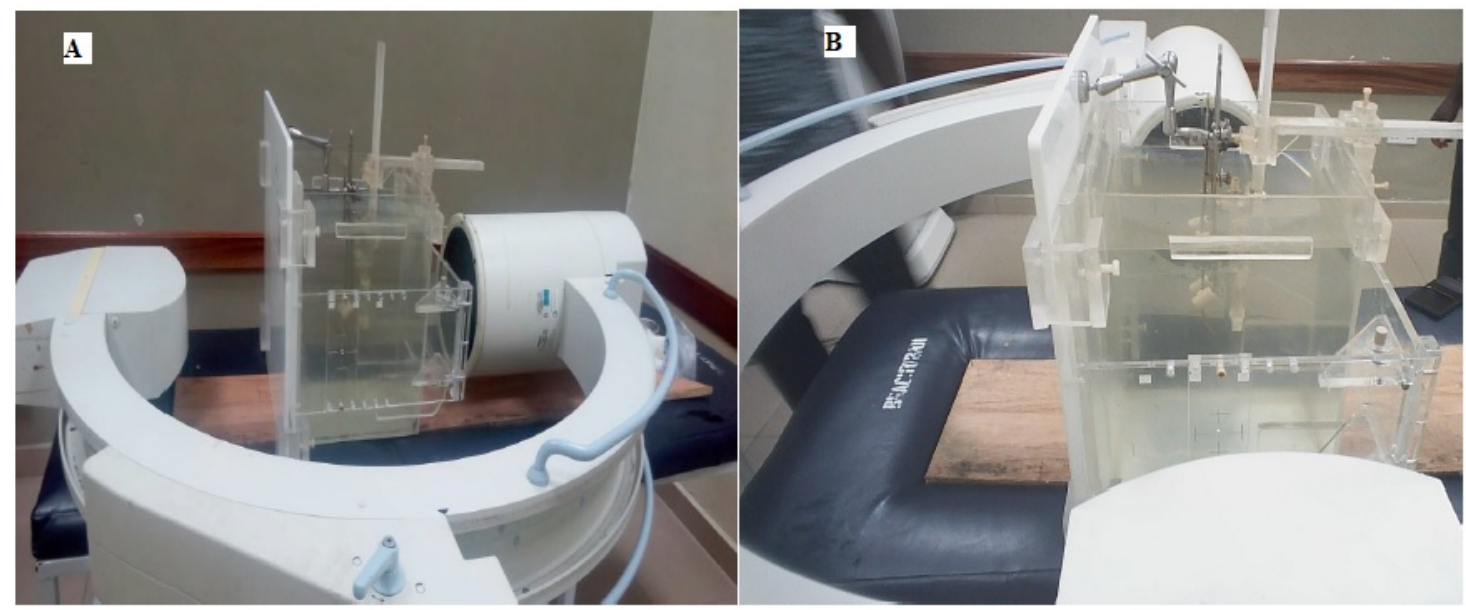

Figure 4 (A and B). Treatment set-up with constructed phantom undergoing imaging with C-arm X-ray machine: A. anterior-posterior orientation and $B$. Lateral orientation 
After the imaging process for an implant, the acquired orthogonal images were saved on a dedicated Universal Serial Bus (USB) drive, and transferred to the HDR-plus TPS (Eckert and Ziegler, Germany) in use for brachytherapy treatment planning by the Department. For tandem and ovoids applicator combinations, doses were prescribed to point A. Whilst for tandem and cylinder applicator combinations doses were prescribed to the surface of the cylinder or to a depth of $0.5 \mathrm{~cm}$. The prescribed doses ranged from 5 to $18.5 \mathrm{~Gy}$. Dose to a marked bladder point for each implant was calculated during the treatment planning process. The brachytherapy treatment plans after completion were exported to the HDR brachytherapy afterloader machine, which is linked to the TPS, and plans executed with the constructed phantom. For the various implants, the film with the radio-opaque object was replaced with a calibrated EBT3 film strip during a treatment delivery process. Also during the treatment delivery processes, it was ensured that the height of the phantom relative to the afterloader machine was adjusted such that there were no excessive bending of the source transfer tubes connected to the various channel indexes and the applicators. This was done to facilitate the smooth and unhindered movement of the dummy source and the real source during treatment delivery. An exposed film was taken through the same process as applied to a film during the film calibration process. For this instant, a point tool was used to define the ROI, which was used to obtain optical densities of exposed films. It was ensured that the point was central to a film (which corresponded with marked bladder point) during the film analyses. The optical densities of films were converted to doses using the correlation equation displayed on the curve in Figure 5. The measured doses were compared to their calculated counterparts by the TPS. In all doses of 85 implants were analyzed.

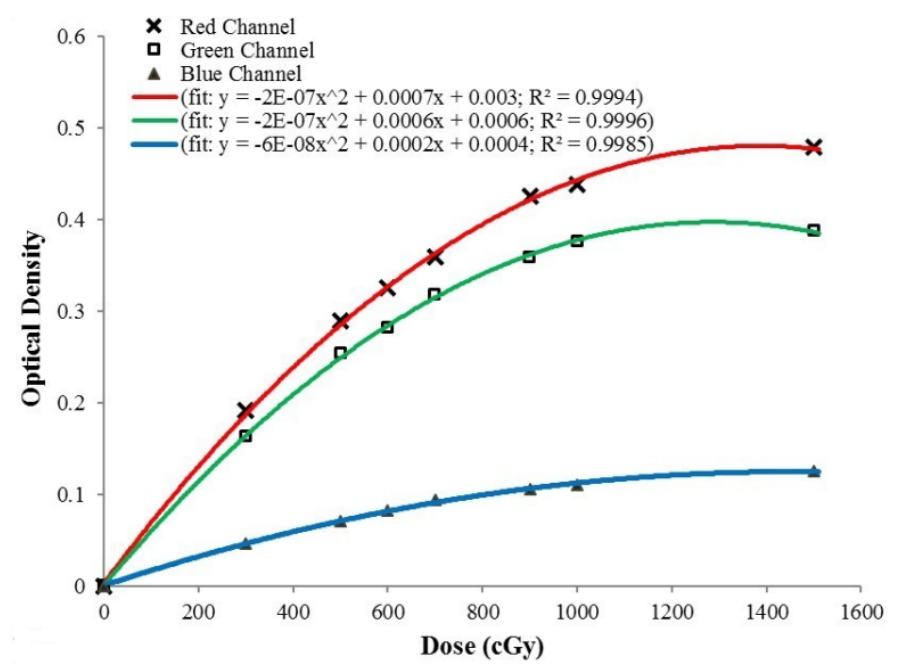

Figure 5. Sensitometry curve of EBT3 film: showing the RBG Colour channels

\section{Results}

The sensitometry curve obtained for the Gafchromic EBT3 during the calibration process is shown in Figure 5. Figure 5 depicts dose as a function of optical density for the various colour channels. Attached to the legion displays on top of the curves are regression analyses of the lines of best fit for the various channels (which are distinguished from each other with appropriate channel colour).

Figure 6 shows the correlation between the net optical density of the red colour channel and radiation dose for the EBT3 film. The dose is expressed as a function of optical density. The correlation equation and its regression, $\mathrm{R}^{2}$ of the line best fit are displayed above the line of best fit in Figure 6.

The measured doses and their corresponding calculated doses generated from the treatment planning system (TPS) are listed in Table 1. The discrepancies between the measured doses and their calculated counterparts which are expressed as percentages of the respectively measured doses are presented. The type of implant or applicators used is also indicated in Table 1.

\section{Discussions}

The overall accuracy of the EBT3 film measurements based on the stipulated uncertainty considerations, and performing error propagations whilst adding the uncertainties in quadrature, was found to be less than $2.5 \%$. The red colour channel of the EBT3 film was found to be the most sensitive to radiation dose than the other colour channels. With reference to this, the red colour channel was chosen as the most appropriate channel for the dose measurements. Though the dose rate of the cobalt 60 source that was used for the EBT3 film calibration was higher than that of the BT source, EBT3 films have been proven to show insignificant dose rate dependence. ${ }^{10}$

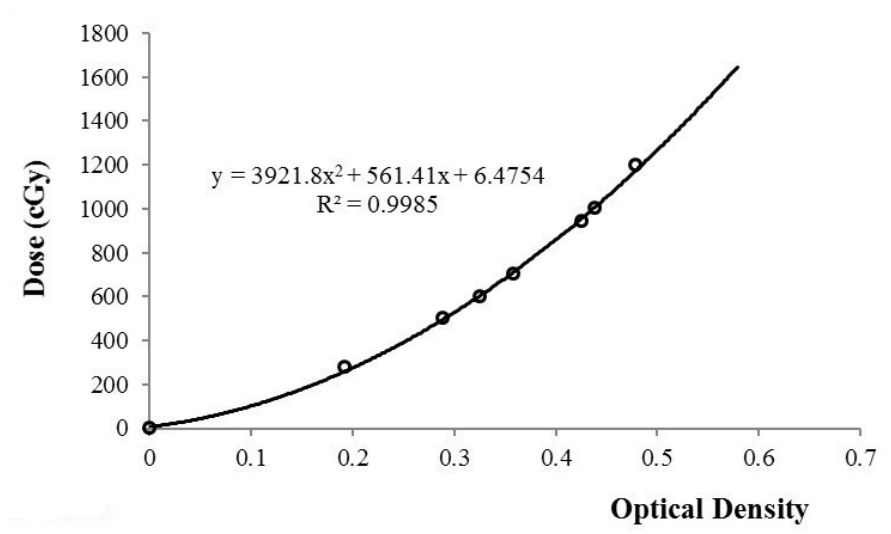

Figure 6. Dose as a function of optical density for Red channel 
Table 1. Comparison of measured dose with EBT3 films and calculated doses with TPS.

\begin{tabular}{|c|c|c|c|c|}
\hline \multirow{2}{*}{$\begin{array}{c}\text { Implant } \\
\#\end{array}$} & \multirow{2}{*}{$\begin{array}{c}\text { Implant } \\
\text { type }\end{array}$} & \multicolumn{2}{|c|}{ "Monitored organ dose (Gy) } & \multirow{2}{*}{$\begin{array}{c}\text { Percentage (\%) } \\
\text { difference between } \\
\begin{array}{l}\text { measured and } \\
\text { calculated dose }\end{array} \\
\end{array}$} \\
\hline & & Measured & Calculated & \\
\hline 1 & VT & 3.45 & 3.57 & 3.48 \\
\hline 2 & VT & 5.67 & 7.00 & 23.46 \\
\hline 3 & VT & 3.07 & 2.65 & -13.68 \\
\hline 4 & VT & 4.07 & 4.00 & -1.72 \\
\hline 5 & TC & 4.55 & 3.20 & -29.67 \\
\hline 6 & TC & 4.05 & 3.20 & -20.99 \\
\hline 7 & VT & 4.50 & 4.27 & -5.11 \\
\hline 8 & VT & 4.00 & 4.27 & 6.75 \\
\hline 9 & TC & 3.52 & 3.23 & -8.24 \\
\hline 10 & TC & 1.86 & 2.47 & 32.80 \\
\hline 11 & VT & 2.17 & 2.11 & -2.76 \\
\hline 12 & VT & 2.38 & 2.32 & -2.52 \\
\hline 13 & VT & 3.05 & 3.57 & 17.05 \\
\hline 14 & VT & 5.25 & 7.00 & 33.33 \\
\hline 15 & VT & 2.57 & 2.65 & 3.11 \\
\hline 16 & VT & 3.57 & 4.00 & 12.04 \\
\hline 17 & VT & 3.02 & 3.23 & 6.95 \\
\hline 18 & $\mathrm{TC}$ & 1.76 & 2.47 & 40.34 \\
\hline 19 & $\mathrm{TC}$ & 2.12 & 2.11 & -0.47 \\
\hline 20 & VT & 2.30 & 2.32 & 0.87 \\
\hline 21 & $\mathrm{TC}$ & 4.49 & 4.50 & 0.13 \\
\hline 22 & $\mathrm{TC}$ & 5.22 & 5.24 & 0.39 \\
\hline 23 & $\mathrm{TC}$ & 7.16 & 7.00 & -2.30 \\
\hline 24 & $\mathrm{TC}$ & 3.49 & 3.65 & 4.56 \\
\hline 25 & $\mathrm{TC}$ & 4.17 & 4.00 & -3.98 \\
\hline 26 & $\mathrm{TC}$ & 3.00 & 3.20 & 6.56 \\
\hline 27 & $\mathrm{TC}$ & 3.05 & 3.20 & 4.82 \\
\hline 28 & TC & 4.15 & 4.27 & 2.85 \\
\hline 29 & $\mathrm{TC}$ & 3.93 & 4.27 & 8.56 \\
\hline 30 & $\mathrm{TC}$ & 3.12 & 3.23 & 3.68 \\
\hline 31 & $\mathrm{TC}$ & 2.37 & 2.47 & 4.25 \\
\hline 32 & $\mathrm{TC}$ & 6.52 & 6.86 & 5.21 \\
\hline 33 & $\mathrm{TC}$ & 2.27 & 2.32 & 1.98 \\
\hline 34 & $\mathrm{TC}$ & 3.41 & 3.57 & 4.63 \\
\hline 35 & $\mathrm{TC}$ & 6.82 & 7.00 & 2.58 \\
\hline 36 & $\mathrm{TC}$ & 2.65 & 2.65 & 0.08 \\
\hline 37 & VT & 4.00 & 4.00 & -0.02 \\
\hline 38 & VT & 3.22 & 3.23 & 0.36 \\
\hline 39 & VT & 2.45 & 2.47 & 0.95 \\
\hline 40 & VT & 2.34 & 2.58 & 10.23 \\
\hline 41 & VT & 4.37 & 3.47 & -20.56 \\
\hline 42 & VT & 3.41 & 3.47 & 1.69 \\
\hline 43 & VT & 6.81 & 7.00 & 2.84 \\
\hline
\end{tabular}

Table 1. Comparison of measured dose with EBT3 films and calculated doses with TPS (continued).

\begin{tabular}{|c|c|c|c|c|}
\hline \multirow{2}{*}{$\begin{array}{c}\text { Implant } \\
\#\end{array}$} & \multirow{2}{*}{$\begin{array}{c}\text { Implant } \\
\text { type }\end{array}$} & \multicolumn{2}{|c|}{ Monitored organ dose (Gy) } & \multirow{2}{*}{$\begin{array}{c}\text { Percentage (\%) } \\
\text { difference betweer } \\
\begin{array}{l}\text { measured and } \\
\text { calculated dose }\end{array}\end{array}$} \\
\hline & & Measured & Calculated & \\
\hline 44 & VT & 6.37 & 6.34 & -0.46 \\
\hline 45 & VT & 5.04 & 6.34 & 25.87 \\
\hline 46 & $\mathrm{TC}$ & 5.34 & 5.54 & 3.78 \\
\hline 47 & $\mathrm{TC}$ & 2.75 & 3.24 & 18.00 \\
\hline 48 & $\mathrm{TC}$ & 4.43 & 4.68 & 5.60 \\
\hline 49 & $\mathrm{TC}$ & 4.99 & 5.32 & 6.70 \\
\hline 50 & $\mathrm{TC}$ & 4.20 & 4.50 & 7.20 \\
\hline 51 & $\mathrm{TC}$ & 2.56 & 2.80 & 9.50 \\
\hline 52 & $\mathrm{TC}$ & 6.56 & 6.84 & 4.20 \\
\hline 53 & $\mathrm{TC}$ & 5.11 & 5.23 & 2.30 \\
\hline 54 & $\mathrm{TC}$ & 6.12 & 6.25 & 2.16 \\
\hline 55 & $\mathrm{TC}$ & 5.63 & 5.34 & -5.23 \\
\hline 56 & $\mathrm{TC}$ & 4.23 & 4.23 & 0.01 \\
\hline 57 & VT & 3.18 & 3.21 & 0.82 \\
\hline 58 & VT & 2.46 & 2.47 & 0.45 \\
\hline 59 & VT & 5.24 & 5.89 & 12.40 \\
\hline 60 & VT & 5.95 & 6.34 & 6.54 \\
\hline 61 & VT & 4.76 & 5.23 & 9.80 \\
\hline 62 & VT & 4.48 & 5.00 & 11.58 \\
\hline 63 & VT & 6.37 & 7.00 & 9.85 \\
\hline 64 & $\mathrm{TC}$ & 4.32 & 4.78 & 10.74 \\
\hline 65 & $\mathrm{TC}$ & 3.19 & 3.69 & 15.62 \\
\hline 66 & $\mathrm{TC}$ & 3.88 & 4.58 & 17.89 \\
\hline 67 & $\mathrm{TC}$ & 3.00 & 3.14 & 4.63 \\
\hline 68 & $\mathrm{TC}$ & 2.75 & 2.89 & 4.98 \\
\hline 69 & $\mathrm{TC}$ & 3.70 & 3.57 & -3.54 \\
\hline 70 & $\mathrm{TC}$ & 4.40 & 4.56 & 3.69 \\
\hline 71 & $\mathrm{TC}$ & 5.37 & 5.49 & 2.22 \\
\hline 72 & $\mathrm{TC}$ & 3.78 & 3.89 & 2.89 \\
\hline 73 & VT & 3.24 & 3.56 & 9.84 \\
\hline 74 & VT & 5.99 & 6.45 & 7.64 \\
\hline 75 & VT & 3.85 & 4.05 & 5.23 \\
\hline 76 & VT & 5.94 & 6.24 & 5.11 \\
\hline 77 & VT & 3.47 & 3.59 & 3.56 \\
\hline 78 & VT & 5.13 & 4.89 & -4.61 \\
\hline 79 & $\mathrm{VT}$ & 2.92 & 2.98 & 2.15 \\
\hline 80 & $\mathrm{TC}$ & 6.81 & 6.48 & -4.87 \\
\hline 81 & $\mathrm{TC}$ & 5.61 & 5.61 & 0.00 \\
\hline 82 & $\mathrm{TC}$ & 4.00 & 4.06 & 1.56 \\
\hline 83 & $\mathrm{TC}$ & 3.73 & 3.82 & 2.35 \\
\hline 84 & $\mathrm{TC}$ & 4.25 & 4.45 & 4.78 \\
\hline 85 & $\mathrm{TC}$ & 3.11 & 3.15 & 1.25 \\
\hline
\end{tabular}

${ }^{*} T C=$ Tandem and cylinder, $V T=$ Tandem and ovoids 
It was therefore reliable to use the determined calibration curve of the EBT3 films to estimate doses for the BT implants. Also, the BT doses were within the range of doses used for the film calibration; making the use of the correlation equation of the line of best fit of the calibration curve very reliable for converting the net optical densities of exposed films into absorbed doses during the measurements. Mean discrepancy of the doses measured compared to those calculated for the presumed bladder points within the fabricated phantom was found to be $7.30 \pm 8.24 \%$ (mean \pm standard deviation), with $56.5 \%$ of the measurements within $\pm 5 \%, 78.8 \%$ within $\pm 10 \%$ and $21.2 \%$ higher than $\pm 10 \%$. These results correlated very well with those of rectum in an in vivo dosimetry study for HDR brachytherapy treatments of vaginal cancer performed by Carrara et al., with three MOSkins dosimeters assembled over a rectal probe. ${ }^{11}$ The percentage difference between measured and calculated doses for presumed bladder points ranged from 29.67 to $40.34 \%$. The extremely high dose discrepancies recorded for some of the implants may be attributed to positional uncertainties between imaging (applicator localization) and treatment delivery. At the onset of treatment delivery, the pseudo-film having a fiducial marker central to it used to mark the bladder point for imaging was replaced with a film, and since the film holder was positioned very close to the applicators in most of the implants there was the likelihood of offsetting the applicators without exercising caution. Notwithstanding this, there could be a shift in the position of the film holder if was loosely secured. The film holder was placed close to the applicators to position a film in the optimal region for favourable detector response that would balance the signal-to-noise ratio (SNR) of the detector, and also to reflect a clinically relevant region for a bladder. The influence of positional uncertainties on the delivered dose was found to be high within this region. ${ }^{12}$ As BT is characterized by sharp dose fall-off with distance, a small unnoticeable change in orientation of the applicators and or the detector holder would lead to significant uncertainties in dose measurements. ${ }^{13}$ Though reimaging directly before treatment delivery may be an alternative way to validate the dose delivery, ${ }^{14}$ there would be the need to exclude doses as a result of the imaging from the measured BT doses. This exercise would be very cumbersome, but due to the sensitivity of the EBT3 film, the dose from the imaging could be ignored as it would cause insignificant fogging of the film if the X-rays exposures are not repeated unnecessarily. Graduated grid templates orthogonal to each other could be placed on the phantom to check the positional uncertainties. Not able to accurately locate a reference point of an exposed film during the film readout with the ImageJ software could also be a contributive factor for the large discrepancies. Finally, excessive bending of the source transfer guide tubes connected to the various applicators could lead to source dwell position uncertainties which would cause the delivered doses to deviate from those planned (or calculated). The influence of dwell position uncertainties on the delivered doses is said to be more pronounced with the tandem than the ovoids. ${ }^{15}$ Since all the implants used had tandem, this phenomena would impact greatly on the measured doses if dwell position errors occurred during the measurements. Neither source calibration errors nor dose calculation algorithm (TG43) of the TPS may be associated with the large discrepancies looking at the results obtained in Table 1. These factors would lead to systemic error in the BT process which would manifest in all measurements. Notwithstanding the influencing factors, the measured doses for the presumed bladder points compared very well to those of other studies. ${ }^{16-19}$ Most of the studies were based on actual clinical measurements.

Major barrier for routine implementation of IVD in BT are as follows: the choice of the appropriate detector to use, the ethical issues raised and the discomfort a patient has to go through with the invasive procedures in which one has to adopt in placing detectors in the patient, the challenge of precise detector positioning to measure the specific organ or tumour doses, the dose calculation becoming more accurate than IVD in most cases, the reasons for the dose disagreements are not always clear, and the added workload for the clinical staff involved with BT procedure. There are also a number of errors that are not detected by safety systems of afterloader BT machines or manual checks. IVD has the potential to complement other QA and quality control (QC) procedures, as part of the greater process of quality management, by providing independent overall verification of the BT treatment delivery. With reference to the above, developments of new IVD systems for BT should involve considerations of time consumption, clinical robustness, and ease of use. The developed and proposed approach comes in handy when considering the above attributes, and may be used for patientspecific QA prior to treatment delivery.

For HDR cervical brachytherapy, there are two radionuclides that can be used, namely; Iridium (Ir) 192 and Cobalt (Co) 60 source. Ir-192 used to be the mostly used for HDR BT. Ir-192 has a half life of 74 days, which is its biggest drawback. In earlier applications the use of Cobalt-60 (Co-60) HDR source was unpopular because the earlier source sizes were larger than Ir-192. ${ }^{20}$ However, in contemporary times, it is now possible to produce miniaturized size of Co-60 radionuclide for HDR applications. Thus, Cobalt-60 has recently emerged as the radionuclide of choice since it has been shown to have identical geometric and dosimetric properties with Ir-192. ${ }^{21}$

\section{Conclusion}

Dose measurements have been carried out with an in-house phantom for various cervical brachytherapy insertions that could be used clinically to assess the accuracy of doses computed by the HDR brachytherapy treatment planning system in use at the study centre. The BT insertions were based on Manchester system, and the doses at specific points within 
the phantom were measured with calibrated gafchromic films for a particular insertion. Doses to the specified points within the phantom were also estimated with the TPS.

The measured doses were compared to their corresponding calculated doses obtained with the TPS. The mean difference between the measured and the TPS calculated doses was $\pm 7.30 \%$ (standard deviation of $\pm 8.24 \%$ ). The uncertainty in this study of $7.30 \pm 8.24 \%$ compared well with other studies. The results of this study provide good evidence for agreement in dose distribution in a definite clinical condition regarding doses to the specific points in the phantom with a non-significant difference in accuracy. The study could therefore be used as a quality assurance tool to evaluate the entire procedures involved with the BT treatment.

\section{Acknowledgement}

The authors wish to thank the National Centre for Radiotherapy and Nuclear Medicine, Korle $\mathrm{Bu}$ Teaching Hospital, for their support during the study.

\section{References}

1. Wilkinson JM, Harris MA, Davidson SE, et al. A retrospective study of bladder morbidity in patients receiving intracavitary brachytherapy as all or part of their treatment for cervix cancer. Br J Radiol. 2003;76:897-903.

2. Garbaulet A, Porter R, Mazeron JJ, et al. The GEC ESTRO Handbook of Brachytherapy. 2002;20:473-480.

3. Viswanathan AN, Thomadsen B. American Brachytherapy Society concensus guidelines for locally advanced carcinoma of the cervix. Part I: General principles. Brachytherapy. 2012;11(1): 33-46.

4. De Leeuw AA, Moerland MA, Nomden C, et al. Applicator reconstruction and applicator shifts in 3D MR-based PDR brachytherapy of cervical cancer. Radiother. Oncol. 2009;93:341-346.

5. Kertzscher G, Rosenfield A, Beddar S, et al. In vivo dosimetry: Trends and prospects for radiotherapy. Br $\mathrm{J}$ Radiol. 2014;87(1041):201-206.

6. International Atomic Energy Agency TRS 398 protocol. Implementation of the international code of practice on dosimetry in radiotherapy (TRS 398): Review of testing results. IAEA-TECDOC, 2010:978-92-0-100610-3.

7. Shima K, Tateoka K, Saitoh Y, et al. Analysis of post-exposure density growth in radiochromic film with respect to the radiation dose. J Radiat Res, 2012;53(2):301-305.

8. Saur S, Frengen J. Gafchromic EBT film dosimetry with flatbed CCD scanner: a novel background correction method and full dose uncertainty analysis. Medical Physics, (2008); 35(7):3094-3101.

9. van Battum LJ, Hoffmans D, Piersma H, et al. Accurate dosimetry with GafChromic EBT film of a 6 MV photon beam in water: what level is achievable? Medical Physics, (2008);35(2): 704-716.

10. Karsch L, Beyreuther E, Burris-Mog T, et al. Dose rate dependence for different dosimeters and detectors. Med. Phys. 2012;39(5):2447-2455.

11. Carrara M, Romanyukha A, Tenconi C, et al. Clinical application of MOSkin dosimeters to rectal wall in vivo dosimetry in gynecological HDR brachytherapy. Physica Medica: European Journal of Medical Physics. 2017;41:5-12.

12. Kertzscher G, Andersen CE, Siebert FA, et al. Identifying afterloading PDR and HDR brachytherapy errors using real-time fibrecoupled Al2O3:C dosimetry and a novel statistical error decision criterion. Radiother. Oncol. 2011;100:456-462.

13. Tanderup K, Beddar S, Andersen CE, et al. In vivo dosimetry in brachytherapy. Medical Physics, 2013;40(7):070902-15.

14. Milickovic N, Mavroidis P, Tselis N, et al. 4D analysis of influence of patient movement and anatomy alteration on the quality of 3D U/S-based prostate HDR brachytherapy treatment delivery. Med. Phys. 2011;38:4982-4993.

15. Reniers B, Landry G, Eichner R, et al. In vivo dosimetry for gynaecological brachytherapy using a novel position sensitive radiation detector: Feasibility study. Med. Phys. 2012;39:1925-1935.

16. Kutcher GJL, Coia M, Gillin WF, et al. Comprehensive QA for radiation oncology: Report of AAPM Radiation Therapy Committee Task Group 40. Med. Phys. 1994;21(4):581-61.

17. Allahverdi M, Sarkhosh M, Aghili M, et al. Evaluation of treatment planning system of brachytherapy according to dose to the rectum delivered. Radiat. Prot. Dosim. 2012;150:312-315.

18. Seymour EL, Downes SJ, Fogarty GB, et al. In vivo real-time dosimetric verification in high dose rate prostate brachytherapy," Med. Phys. 2011;38:4785-4794.

19. Waldhäusl C, Wambersie A, Potter R, and Georg D. In-vivo dosimetry for gynaecological brachytherapy: Physical and clinical considerations. Radiother. Oncol. 2005;77:310-317.

20. Chao KS, Perez CA, Brady LW. Physics and Dosimetry of High-Dose-Rate Brachytherapy in Radiation Oncology Management Decisions (2nd Edition). Lippincott Williams \& Wilkins. Philadelphia. 2002;89-94.

21. Ballester F, Granero D, Perez-Calatyud J, Casal E, Agramunt S, Cases R. Monte Carlo dosimetric study of the BEBIG Co-60 HDR source. Phys Med Biol. 2005;50:N309-16. 